\title{
Progressive Failure Analysis of Composite Stiffened Panels
}

\author{
Brett A. Bednarcyk ${ }^{1}$ \\ Ohio Aerospace Institute, Cleveland, OH 44142 \\ Phillip W. Yarrington ${ }^{2}$, and Craig S. Collier ${ }^{3}$ \\ Collier Research Corp., Hampton, VA 23669 \\ Steven M. Arnold ${ }^{4}$ \\ NASA Glenn Research Center, Cleveland, OH 44135
}

\begin{abstract}
A new progressive failure analysis capability for stiffened composite panels has been developed based on the combination of the HyperSizer stiffened panel design/analysis/optimization software with the Micromechanics Analysis Code with Generalized Method of Cells (MAC/GMC). MAC/GMC discretizes a composite material's microstructure into a number of subvolumes and solves for the stress and strain state in each while providing the homogenized composite properties as well. As a result, local failure criteria may be employed to predict local subvolume failure and the effects of these local failures on the overall composite response. When combined with HyperSizer, MAC/GMC is employed to represent the ply level composite material response within the laminates that constitute a stiffened panel. The effects of local subvolume failures can then be tracked as loading on the stiffened panel progresses. Sample progressive failure results are presented at both the composite laminate and the composite stiffened panel levels. Deformation and failure model predictions are compared with experimental data from the World Wide Failure Exercise for AS4/3501-6 graphite/epoxy laminates.
\end{abstract}

\section{Introduction}

Progressive failure occurs when a structure experiences a significant amount of damage prior to final failure. The life of the structure is therefore not accurately represented by the point at which failure initiates, rather, failure progresses from initiation to final failure in some way. Clearly, in order to predict the life of such a structure, a methodology that tracks the failure progression is needed. Herein a new progressive failure capability for composite stiffened panels and laminates is presented and demonstrated. It has been enabled via the combination of Collier Research Corporation's HyperSizer ${ }^{1}$ stiffened panel design software with NASA Glenn Research Center's Micromechanics Analysis Code with Generalized Method of Cells (MAC/GMC) ${ }^{2,3}$. MAC/GMC can provide the ply level composite material properties to HyperSizer, from which HyperSizer can determine laminate and panel level properties. Further, because MAC/GMC localizes to the level of the fiber and matrix constituents, micro scale failures can be predicted given ply level stresses and strains from HyperSizer. The new ability of HyperSizer to apply loading to a stiffened panel incrementally (i.e., time-dependent loading) has now enabled progressive failure analysis based on local failures predicted within MAC/GMC, along with the elimination of the stiffness contribution of the failed regions within the fiber and matrix constituents.

\footnotetext{
${ }^{1}$ Senior Scientist, 22800 Cedar Point Rd., Cleveland, OH 44142, bednarcyk@oai.org, AIAA Member.

${ }^{2}$ Senior Research Engineer, phil.yarrington@hypersizer.com, AIAA Member.

${ }^{3}$ Senior Research Engineer, craig.collier@hypersizer.com, AIAA Senior Member.

${ }^{4}$ Senior Research Engineer, 21000 Brookpark Rd., Cleveland, OH 44135, steven.m.arnold@nasa.gov.
} 


\section{Time-Dependent Loading}

The extension of HyperSizer to apply time-dependent loading enables the software to step through a thermo-mechanical loading profile on a stiffened composite structure rather than considering loads as just static points at which an analysis is performed. Now, when coupled with HyperSizer's new MAC/GMC micromechanics capabilities, a MAC/GMC analysis is performed for each ply in each stiffened panel component at each step of the applied global loading. Local damage and failures can then occur at the level of the fiber and matrix constituents throughout the panel and affect the local ply stiffness. The reduced stiffness of the plies in turn affects the extensional, coupling, and bending (ABD) stiffness matrices of their laminates, which affects the response of the panel as a whole. Thus, progressive damage and failure analysis of composite laminates and stiffened panels can now be performed within HyperSizer, which represents a major step forward for the software analysis capabilities. In addition, a new panel-level global failure criterion has been developed and implemented in order to predict overall failure as a result of accumulated local damage. Finally, a methodology for determining design margins of safety, quantities critical to the core HyperSizer capabilities, based on progressive failure analysis, has been developed and implemented.

HyperSizer's new time-dependent capabilities allow the code to apply loading in an incremental fashion, linearly stepping to a given load level rather than applying the load all at once. The time stepping procedure is outlined as,

1) Zero all stress and strain fields

2) Loop over the plies of the stiffened structure, calling MAC/GMC at each ply to obtain effective elastic properties. These properties take into account any damage that has occurred during any previous step.

3) Integrate the local ply properties to calculate the global ABD matrices for the stiffened structure.

4) Apply loads (global $\mathrm{N}_{\mathrm{x}}, \mathrm{N}_{\mathrm{y}}$, etc.) and determine global response $(\varepsilon, \kappa$, etc.).

5) Resolve ply-level strains from global strains and curvatures.

6) Pass ply-level strains to MAC/GMC. MAC/GMC calculates subcell local fields, determines damage (if any), and returns margins of safety for given ply strains to HyperSizer.

7) Return to step 2 for the next time step, where any damage occurring during the present step will be reflected in the ABD matrix during the next step.

In the context of time-dependent analysis, HyperSizer's ability to consider multiple load cases now enables consideration of a history-dependent loading profile. A load case is a panel-level set of loading conditions consisting of $\mathrm{N}_{\mathrm{x}}$ or $\varepsilon_{\mathrm{x}}, \mathrm{N}_{\mathrm{y}}$ or $\kappa_{\mathrm{y}}, \mathrm{N}_{\mathrm{xy}}$ or $\kappa_{\mathrm{xy}}, \mathrm{M}_{\mathrm{x}}$ or $\kappa_{\mathrm{x}}, \mathrm{M}_{\mathrm{y}}$ or $\kappa_{\mathrm{y}}, \mathrm{M}_{\mathrm{xy}}$ or $\kappa_{\mathrm{xy}}, \mathrm{Q}_{\mathrm{x}}, \mathrm{Q}_{\mathrm{y}}$, temperature change from reference, and through-thickness temperature gradient. HyperSizer traditionally has considered each such load case separately, determining margins of safety for each. With the new time-dependent capabilities, each load case has an associated time and a time step size, and HyperSizer treats the load cases as a load profile, as shown in Fig. 1. The load cases thus form a number of points, and HyperSizer steps linearly from zero load to the first load point, then to the second, and so on. While stepping to a load point, local damage can potentially occur, thus making the panel response history dependent. As shown in Fig. 1, this potential path dependence could cause the panel response to the two depicted load profiles to be different, despite the fact that all load points are identical. In contrast, HyperSizer's previous time-independent capabilities would have predicted the panel response to both load profiles as identical. 

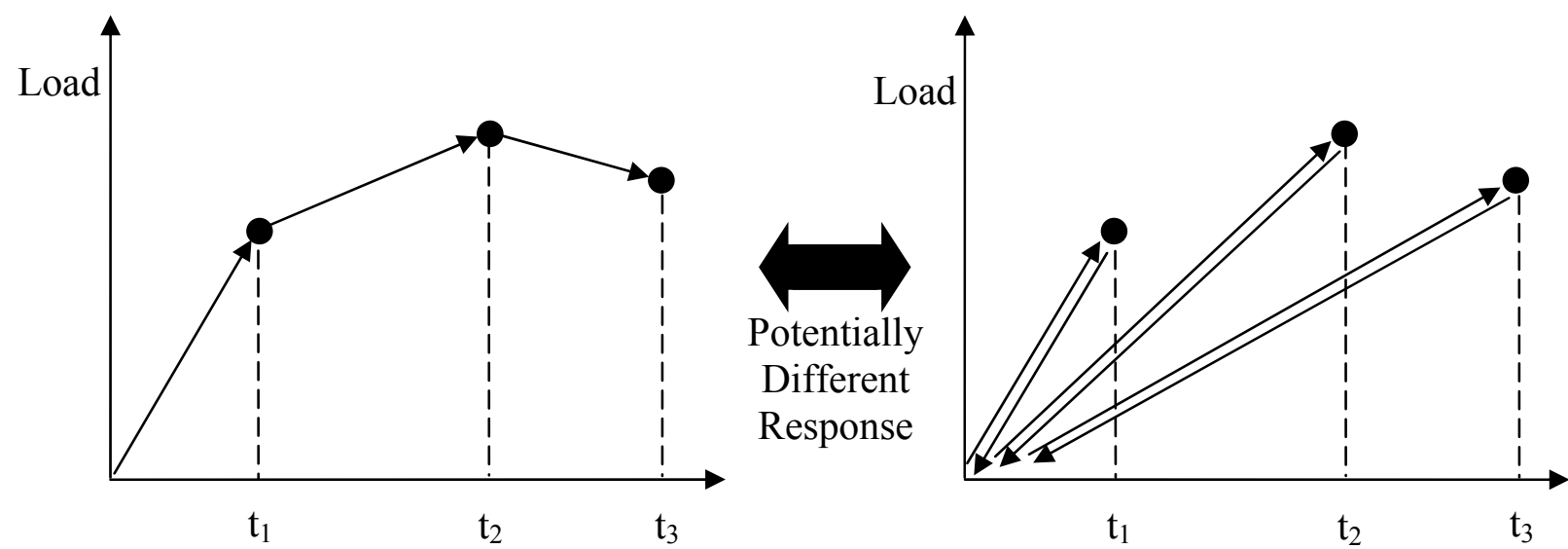

Fig. 1. HyperSizer's new time-dependent capabilities transform its existing load cases into a load profile through which the code steps. The two profiles shown, consisting of identical load points, now potentially yield different panel responses.

\section{Panel Level Failure Criterion and Margin of Safety}

In order to predict overall panel failure during a time-dependent analysis, and thus determine a margin of safety for load levels below failure, a panel level failure criterion is needed. In the case of static HyperSizer panel analyses, ply-level failure theories are used to calculate the panel margin of safety. That is, the panel stresses and strains are localized to determine ply-level stresses and strains, which are then used in anisotropic failure theories such as the maximum stress criterion and the Tsai-Wu criterion. This type of static analysis considers panel failure to occur upon failure of the first ply. In the new time-dependent HyperSizer analyses, a ply may damage considerably before its contribution to the panel is no longer significant, and several plies may damage completely (i.e., fail) before final failure of the panel. Clearly, a traditional failure theory, based on panel- or ply-level stresses or strains, is not appropriate in this context. Rather, a criterion that evaluates the panel's global ability to support further loading is needed. Candidate criteria related to the rate of change of the panel strain energy density and to the trace of the panel ABD matrix were considered and evaluated. It was determined that the strain energy density rate experiences a disturbance (i.e., large drop) upon each local failure within the panel, and was thus not well-suited, while the trace of the ABD matrix did not experience a large change as a given laminate failed, even when the overall apparent stiffness of the laminate was reduced to near zero. The trace of the inverse of the panel ABD matrix, on the other hand, did exhibit large changes during progressive failure, without the unwanted disturbances. This quantity represents a measure of the panel's compliance, and includes normal and shear extensional and bending contributions. After several trials of this procedure, an increase of an order of magnitude in the trace of the panel inverse ABD matrix has been chosen as the failure criterion in HyperSizer. Upon fulfillment of this criterion, the time stepping within HyperSizer stops, and the highest previously achieved load level is chosen as the panel or laminate ultimate load.

A sample laminate progressive failure response illustrating the implemented trace inverse ABD failure criterion is given in Fig. 2. In this quasi-isotropic laminate example, the behavior of each ply is governed by a MAC/GMC micromechanics analysis of a repeating unit cell (see Fig. 9). Damage accumulates as the subcells, which comprise the repeating unit cell, reach a stress or strain state that fulfills a failure criterion. In the present example, the maximum stress criterion was employed on this local level. As shown in Fig. 2, damage initiates at an applied midplane strain of 0.005, when a limited number of subcells reach their failure stress. However, it is not until a midplane strain of 0.01 that a large 
drop in the force resultant is observed. At this point, the trace of the laminate inverse ABD matrix increases by a factor of 4.8, and as Fig. 2 shows, the laminate force resultant continues to rise as the applied midplane strain increases. It is not until the applied midplane strain reaches a value of 0.02 that the inverse ABD increases by an order of magnitude from its original value, signaling global failure in HyperSizer. Clearly, at this point the laminate cannot support any additional loading. HyperSizer then uses the highest achieved load level, $\mathrm{N}_{\mathrm{x}}=4.85 \mathrm{kips} / \mathrm{in}$. in the example, as the failure load for the laminate or panel. It should be noted that the time step size, as well as the repeating unit cell discretization (see Fig. 9) will have an effect on the progressive failure results.

Predicting overall failure based on progressive micro scale failure is a new capability for HyperSizer, but for full implementation, the new capability must automatically calculate a margin of safety (MOS) based on a user specified load. With the new HyperSizer time-dependent capabilities, users can now specify an entire load profile (i.e., load vs. time points, see Fig. 1), but MOS values must be calculated for each specified load level. The MOS is defined as:

$$
\text { MOS }=\frac{\text { Allowable Load }}{\text { Actual Load }}-1
$$

It is a measure of how close the actual (i.e., specified) load is to the allowable (i.e., failure) load. If the actual load is small compared to the allowable load, the MOS will be large, indicating a safe load. If the actual load is at or below the allowable load, the MOS will be zero or negative, indicating an unsafe load. In the context of time-dependence, if the user specifies load levels that are below the allowable load, a question arises as to how to determine the MOS since the allowable load will not be known. That is, if a specified load is below the failure load, HyperSizer will reach that load without detecting final failure, so the allowable load will not be known. The procedure developed and implemented within HyperSizer for determining time-dependent MOS is depicted in Fig. 3. If a user-specified load point is reached without reaching overall failure, HyperSizer stores the information at that point, and then continues loading until overall failure is predicted. This then establishes the allowable load, and the MOS for that load point can be calculated from Eq. (1). HyperSizer then returns to the specified load point and continues loading along the path to the next load point. If failure is not detected before this next load point is reached, HyperSizer again stores the information at the point and continues loading. However, as shown in Fig. 3, the loading continues not along the path between the load points, but along the path defined by the origin and load point. This then gives a true measure of how close the specified load point is to failure and accounts for any previous damage that has occurred in reaching the specified load point. If HyperSizer predicts overall failure along the path between two specified load points (as shown in Fig. 3 between points 3 and 4), then the negative MOS is calculated based on how far along the path the panel progressed before the overall failure. It should be noted that the load points are, in general, multi-axial, and thus Fig. 3 is just a schematic representation of the procedure.

\section{Verification and Validation Examples}

HyperSizer time-dependent progressive failure analysis results were verified by considering a simplified geometry (a laminate rather than a stiffened panel) via comparison to MAC/GMC timedependent results. Note that the HyperSizer panel-level time-dependent progressive failure analysis capabilities are unique and thus not able to be verified in this fashion. A verification example involving progressive failure of a fictional complex symmetric laminate consisting of doubly- and triply-periodic layers is shown in Fig. 4. Results for two different time step sizes are shown and in both cases, MAC/GMC and HyperSizer predictions are virtually identical. The example also indicates the time point at which the overall failure criterion employed in HyperSizer (based on the trace of the inverse of the ABD matrix) is fulfilled, thus stopping the code's execution. Fig. 5 shows another verification case for a 


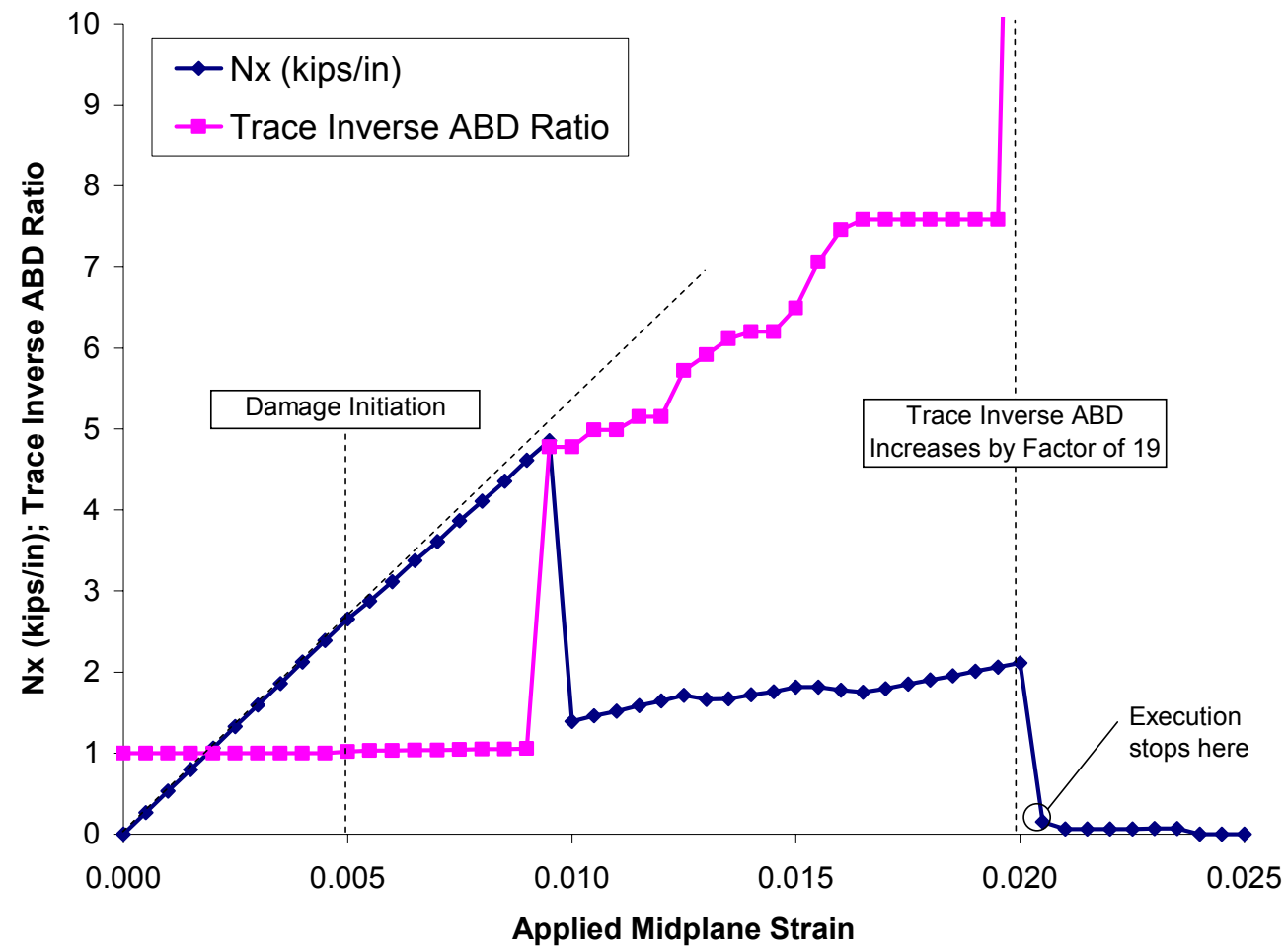

Fig. 2. HyperSizer progressive failure simulation of a quasi-isotropic graphite epoxy laminate; force resultant vs. applied midplane strain and trace inverse ABD ratio (trace inverse ABD / original trace inverse ABD) vs. applied midplane strain. An increase of 10 times of the trace of the inverse ABD from its initial (undamaged) value was chosen as the failure criterion for cessation of the time stepping in HyperSizer. HyperSizer then chooses the highest previous load level as the ultimate load.

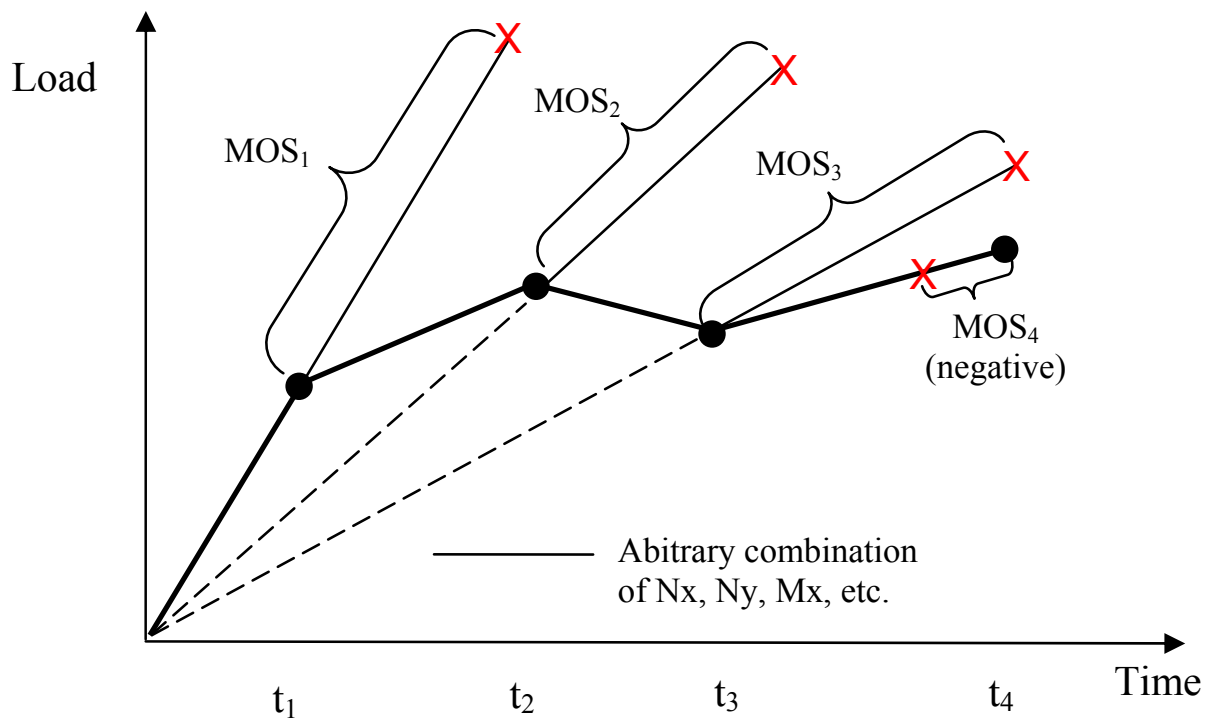

Fig. 3. The procedure developed and implemented within HyperSizer for determining timedependent margins of safety (MOS) for each specified load point involves automatically stepping along a path connecting the origin with the load point until overall failure occurs. 


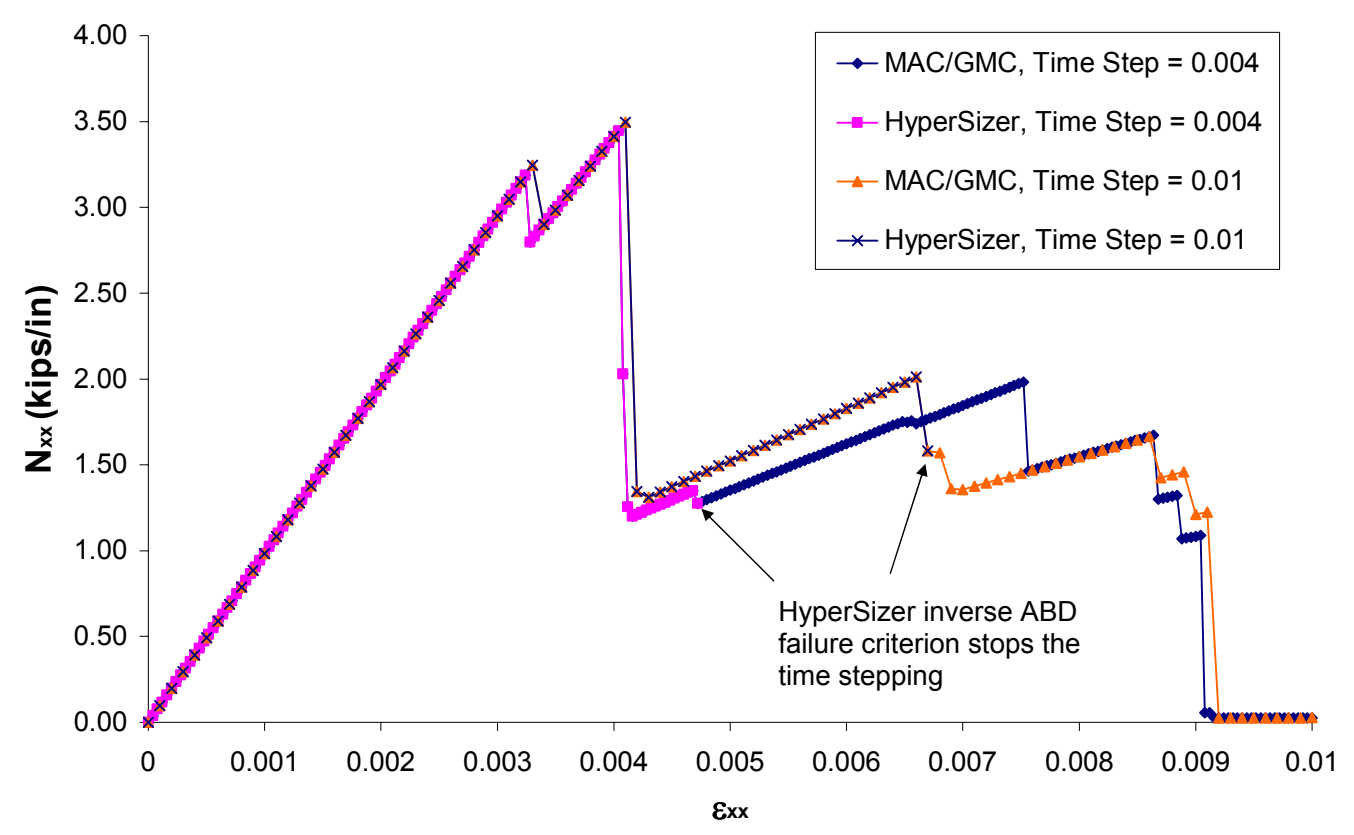

Fig. 4. Comparison of HyperSizer to MAC/GMC for the predicted progressive failure response of a fictional symmetric laminate containing both doubly-periodic and triply-periodic composite layers. Results are nearly identical.

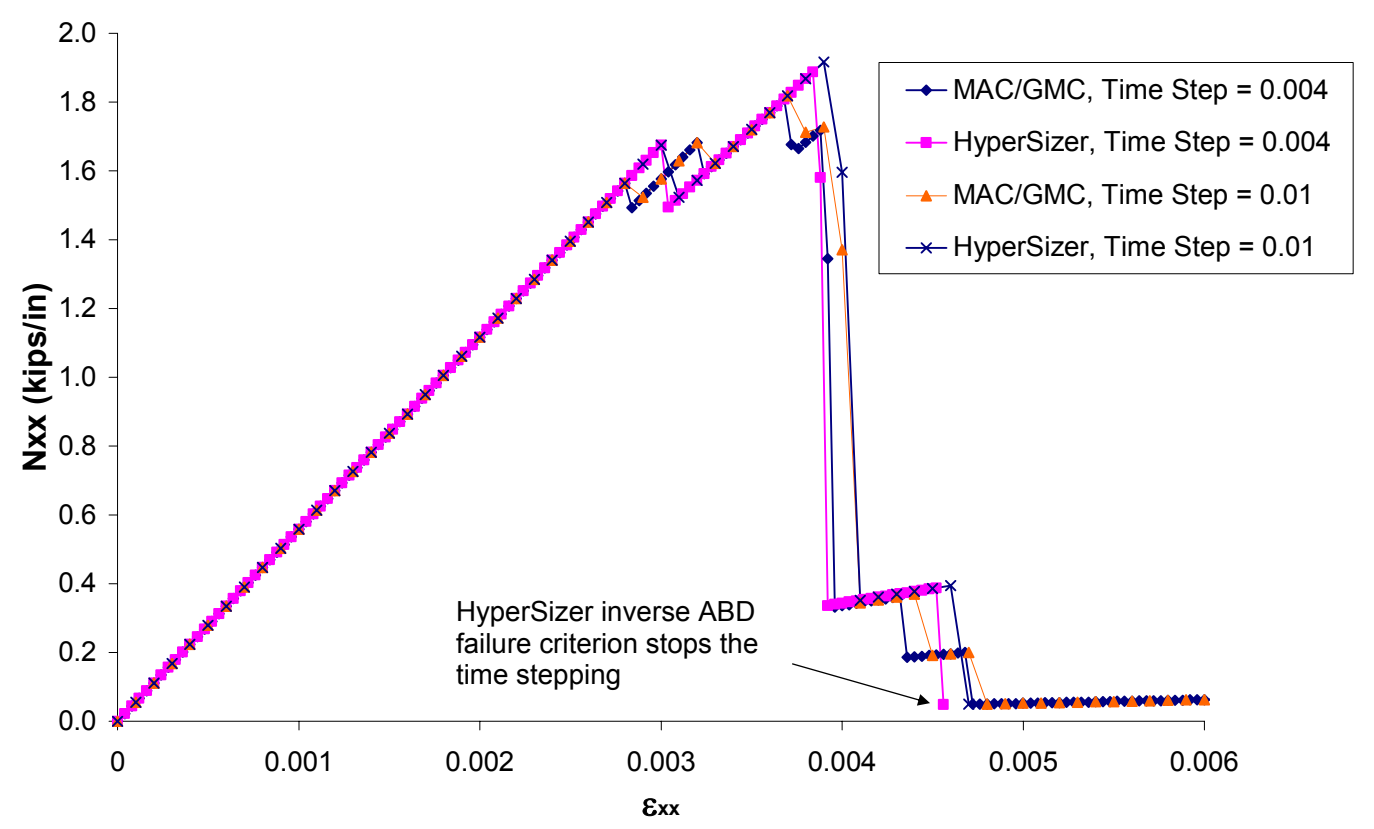

Fig. 5. Comparison of HyperSizer to MAC/GMC for the predicted progressive failure response of a fictional asymmetric laminate. The differences are due to the fact that MAC/GMC tracks the damage at two points per ply, while HyperSizer only employs one. This has an effect when, as in this asymmetric case, bending occurs. 
similarly complex asymmetric laminate, which due to the asymmetry, experiences bending. Results for two different time step sizes are again shown, and the MAC/GMC and HyperSizer results are very similar. Slight differences occur in the vicinity of local failures due to the fact that MAC/GMC tracks damage at two points per ply, while HyperSizer tracks damage at only one.

The new HyperSizer time-dependent progressive failure analysis capabilities were applied to analyze a composite laminate studied as part of the World Wide Failure Exercise (WWFE) ${ }^{4}$ The WWFE provided ply level properties for four polymer matrix composites and invited leading composite failure theory researchers to predict the deformation and strength of several laminate lay-ups without prior access to the laminate level results. In subsequent papers, the WWFE compared the theoretical predictions with experimental results 5 . The WWFE results have thus become a standard for polymer matrix composite laminate failure analysis. The WWFE tests were performed on tubular specimens, wherein the loading was applied as combined internal pressure and axial load (load control). The analyses performed herein, like those included within the WWFE, treat the laminates as flat utilizing HyperSizer's laminate analysis based on classical lamination theory.

In Figs. 6 and 7, the HyperSizer-MAC/GMC time-dependent progressive failure predictions are compared with the biaxial WWFE experimental results for a quasi-isotropic [0/ $\pm 45 / 90]_{\mathrm{s}}$ AS4 graphite/3501-6 epoxy composite. The applied stress ratios $\left(S R=\sigma_{x x}: \sigma_{y y}\right)$ are 20:1 and 2:1, respectively. At the local subcell level within MAC/GMC, the maximum stress criterion was employed to govern local failure. The results show that the new HyperSizer time-dependent progressive failure capabilities are able to capture the non-linear deformation response (due to local damage) of the laminates quite accurately (with the stiffness loss over predicted somewhat in the case of the 20:1 SR). In addition, HyperSizer, utilizing the inverse ABD criterion to stop execution, provides a good, slightly conservative prediction of the laminate strength. Fig. 8 shows compares the full [0/ $\pm 45 / 90]_{\mathrm{s}}$ AS4/3501-6 failure envelope predicted by the new HyperSizer time-dependent progressive failure capabilities with the WWFE experimental results. The HyperSizer predictions for final failure as well as initiation are plotted, along with the corresponding WWFE experimental results. Also plotted for comparison are first ply failure envelopes predicted by other leading ply-level failure theories. These results clearly indicate the improvement represented by HyperSizer's new time-dependent capabilities (when functioning in conjunction with a MAC/GMC micro scale analysis). Failure initiation can now be predicted by HyperSizer, and as shown in Fig. 8, the failure initiation surface is close to the failure predictions made using most ply-level theories. More importantly, the final failure envelope predicted by HyperSizer is excellent agreement with the WWFE experimental results and far superior to the ply-level theory envelopes.

The next step, as shown schematically in Fig. 9, is to perform analysis of a stiffened panel. A Tstiffened panel, like that shown in Fig. 9, composed of a [0/ $\pm 45 / 90]_{\mathrm{s}}$ AS4/3501-6 facesheet and a [0/ $\pm 45 / 90]_{\mathrm{S}}$ AS4/3501-6 T-stiffener, has been analyzed using HyperSizer. Deformation results for a stress ratio of 1:0 are shown in Fig. 10. Note the presence of nonlinearity due to local damage of the epoxy matrix. Fig. 11 shows the full predicted failure envelope for the T-stiffened panel for both damage initiation and final failure. Envelopes predicted by other ply-level failure theories are once again plotted for comparison. Comparing the panel failure envelopes (Fig. 11) to the laminate failure envelopes (Fig. 8), the most notable similarity is the much larger size of the HyperSizer progressive failure envelope in quadrant I compared to the ply level failure criteria envelopes. The ply level failure criteria envelopes once again agree well with the HyperSizer damage initiation envelope in quadrant I. However, in quadrant 3, the stiffened panel HyperSizer damage initiation envelope is far inside the ply level and HyperSizer ultimate failure envelopes. Because experimental data are not available for this T-stiffened panel, it is not currently possible to comment on the accuracy of these HyperSizer progressive failure analysis results. 


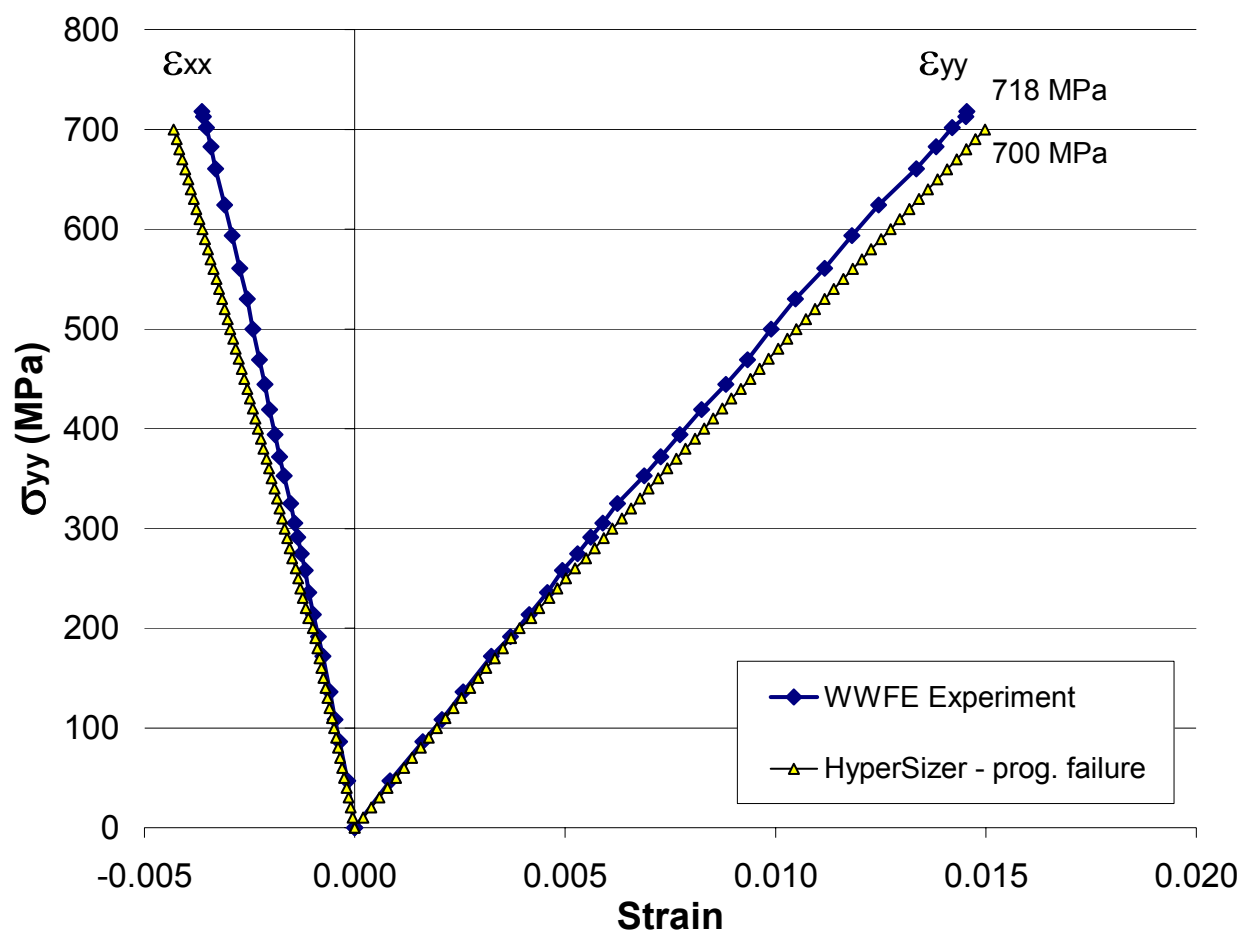

Fig. 6. Comparison of time-dependent HyperSizer progressive failure deformation predictions with WWFE biaxial experimental results for $[0 / \pm 45 / 90]_{\mathrm{s}} \mathrm{AS4/3501-6}$ with $\mathrm{SR}=20: 1$.

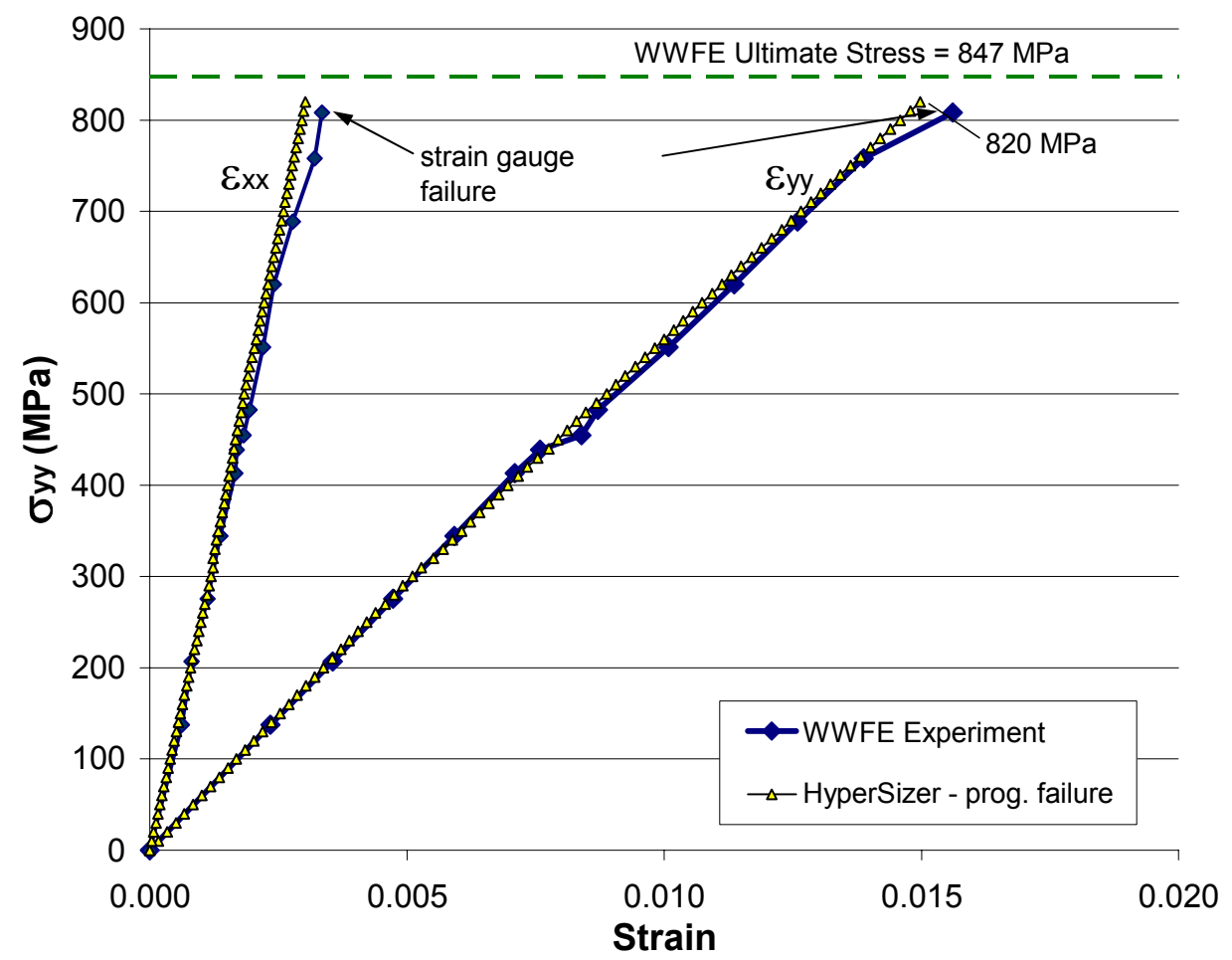

Fig. 7. Comparison of time-dependent HyperSizer progressive failure deformation predictions with WWFE biaxial experimental results for $[0 / \pm 45 / 90]_{s}$ AS4/3501-6 with $S R=2: 1$. Note that in the experiment, strain gauge failure occurred prior to reaching the ultimate stress of $847 \mathrm{MPa}$. 


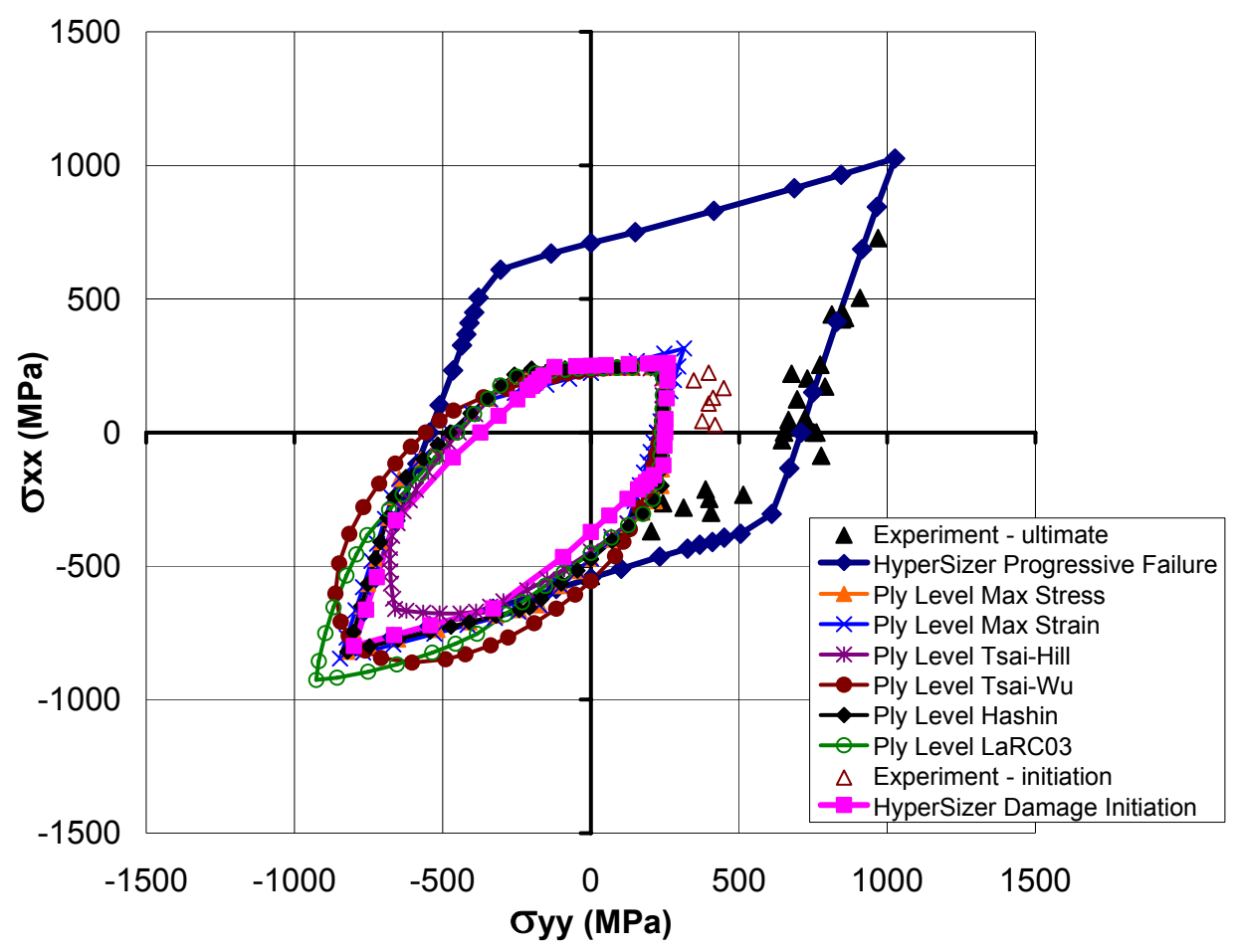

Fig. 8. Comparison of time-dependent HyperSizer progressive failure deformation predictions with WWFE experimental results for the $[0 / \pm 45 / 90]_{s}$ AS4/3501-6 failure envelope. Predictions made with other ply-level failure criteria are plotted as well.

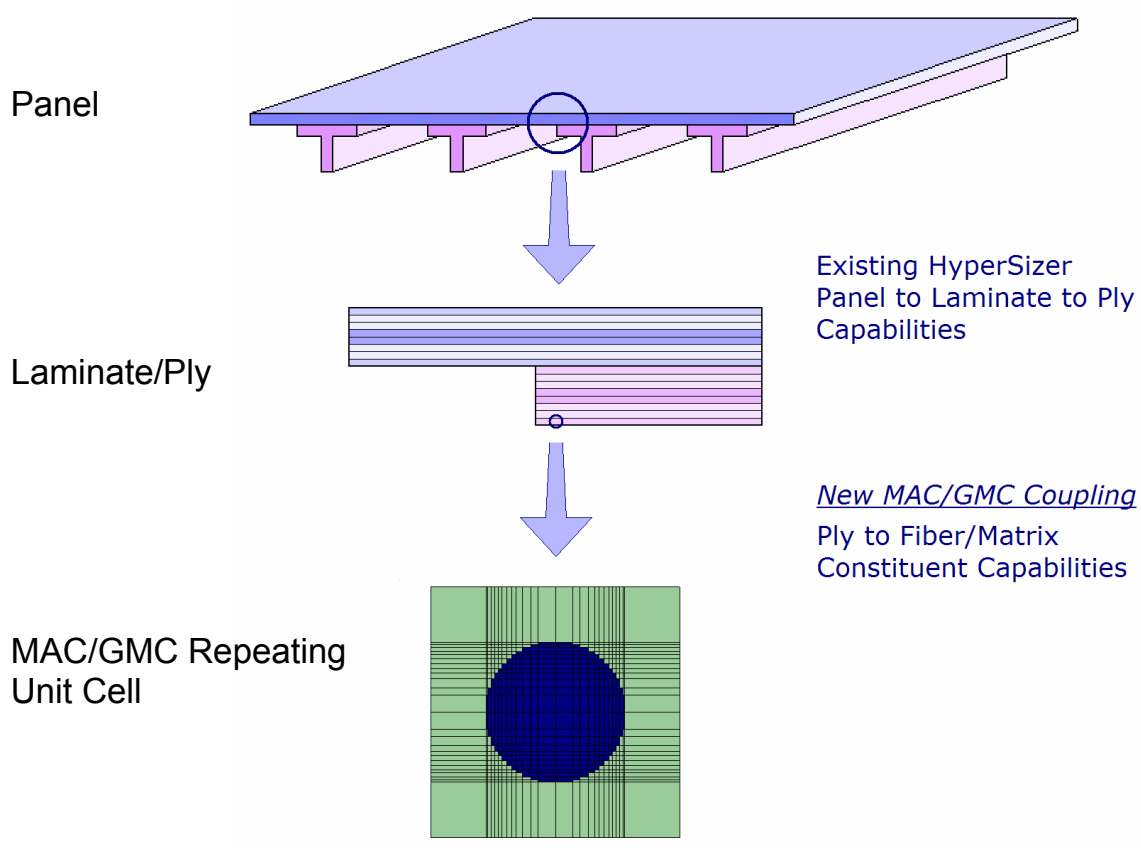

Fig. 9. Schematic of the T-panel to ply to micromechanics simulations now available within HyperSizer in the context of time-dependence and progressive failure. 


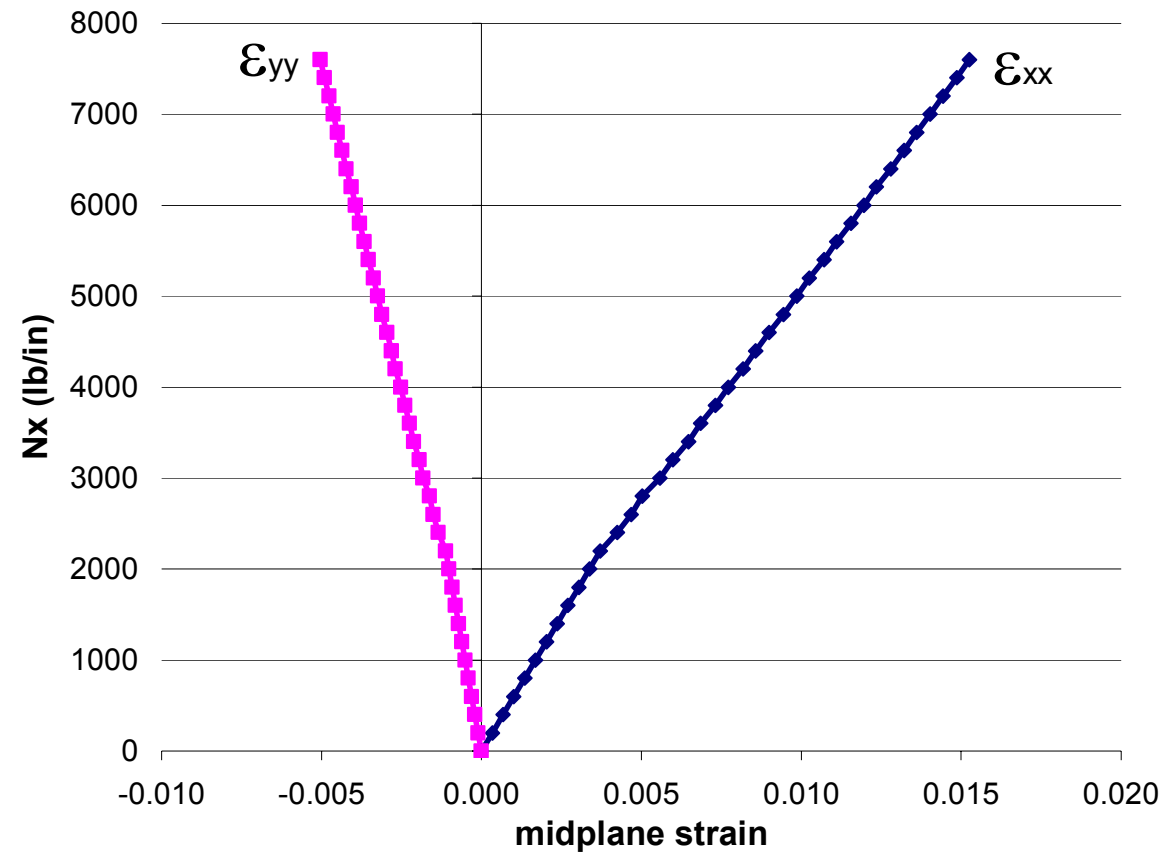

Fig. 10. Time-dependent HyperSizer progressive failure deformation predictions for a $[0 / \pm 45 / 90]_{\mathrm{s}}$ AS4/3501-6 T-stiffened panel, $S R=1: 0$.

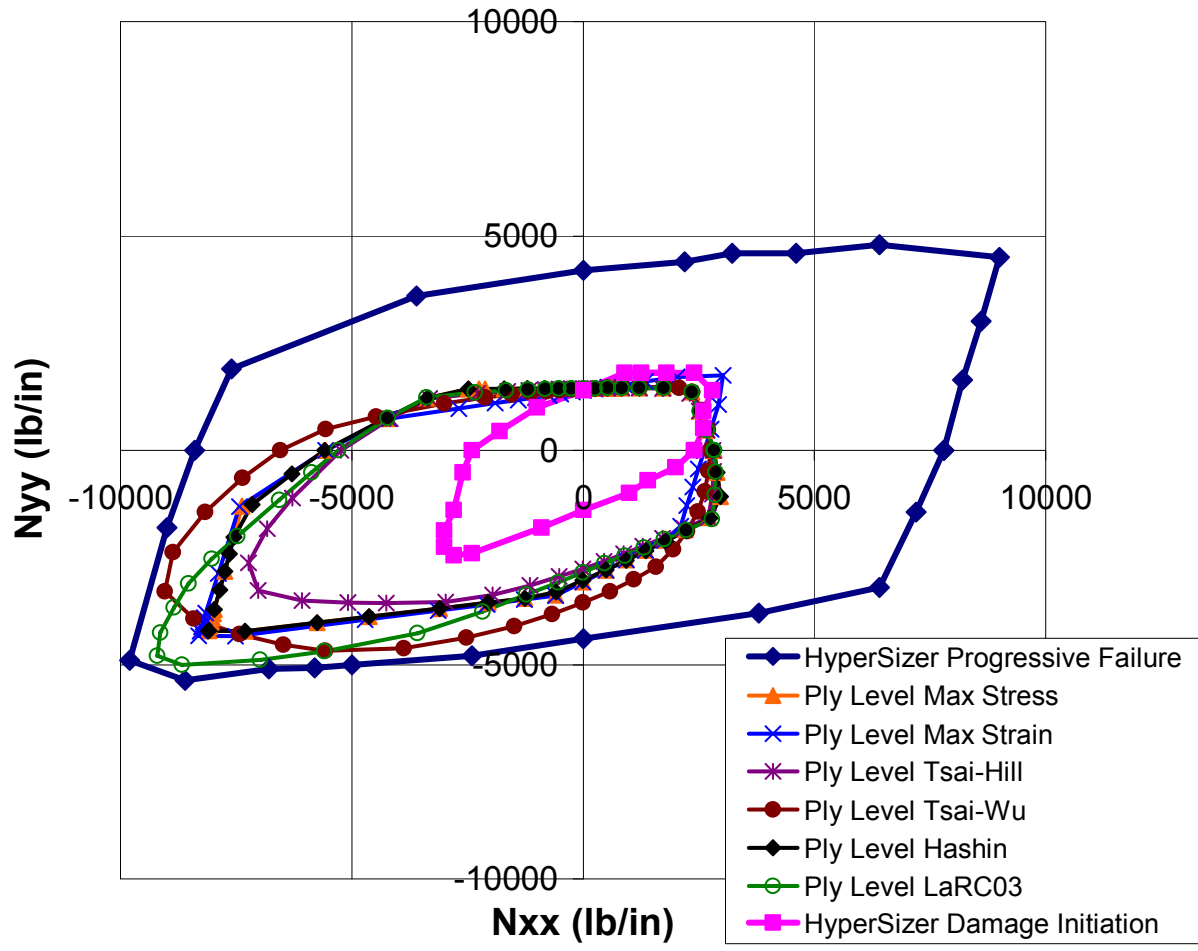

Fig. 11. Time-dependent HyperSizer progressive failure predictions for the failure envelope of a $[0 / \pm 45 / 90]_{s}$ AS4/3501-6 T-stiffened panel. Predictions made with other ply-level failure criteria are plotted as well. 


\section{Conclusion}

A new analysis capability that enables the prediction of failure of composite stiffened panels and laminates based on progressive failure has been presented. Through the combination of the MAC/GMC micromechanics software and the HyperSizer stiffened panel analysis software, local failures within the fiber and matrix constituents can be tracked and their effects on the global structural response predicted. A new method for predicting global panel failure based on an order of magnitude increase in the panel inverse ABD matrix, as well as a method for determining time-dependent panel margins of safety, have been developed and implemented. The new capabilities have been verified and validated through comparison with WWFE results for composite laminates and exhibit good agreement with the experimental results.

\section{References}

1. Collier Research Corp. (1998) HyperSizer Structural Sizing Software. Hampton, VA.

2. Bednarcyk, B.A. and Arnold, S.M. (2002a) "MAC/GMC 4.0 User's Manual Volume 2: Keywords Manual" NASA-TM-212077/Vol 2.

3. Bednarcyk, B.A. and Arnold, S.M. (2002b) "MAC/GMC 4.0 User's Manual Volume 3: Example Manual" NASA-TM-212077/Vol 3.

4. Soden, P.D., Hinton, M.J., and Kaddour, A.S. (1998) "Lamina Properties, Lay-Up Configurations and Loading Conditions for a Range of Fibre-Reinforced Composite Laminates" Composites Science and Technology 58, 1011-1022.

5. Soden, P.D., Hinton, M.J., and Kaddour, A.S. (2002) "Biaxial Test Results for Strength and Deformation of a Range of E-Glass and Carbon Fibre Reinforced Composite Laminates: Failure Exercise Benchmark Data" Composites Science and Technology 62, 1489-1514. 